\title{
On cusps and flat tops
}

\author{
Neil Dobbs
}

October 29, 2018

\begin{abstract}
Non-invertible Pesin theory is developed for a class of piecewise smooth interval maps which may have unbounded derivative, but satisfy a property analogous to $C^{1+\epsilon}$. The critical points are not required to verify a non-flatness condition, so the results are applicable to $C^{1+\epsilon}$ maps with flat critical points. If the critical points are too flat, then no absolutely continuous invariant probability measure can exist. This generalises a result of Benedicks and Misiurewicz.
\end{abstract}

\section{Introduction}

The ergodic theory of real one-dimensional dynamical systems has been a topic of intense study in recent decades. Especially, much progress has been made for smooth maps of the interval with non-flat critical points $(9,22,6$, 7, to cite a few), and the theory underlying such dynamical systems is now well-understood. In this work we aim to develop some aspects of the theory beyond the smooth, non-flat setting. The principal results are Theorems 10 5, 6 and 16.

\subsection{Smooth maps with flat tops}

For smooth maps without a non-flatness condition on the critical points, results are limited. Benedicks and Misiurewicz (3] ) showed that under a non-recurrence condition, unimodal maps with negative Schwarzian derivative have an ergodic, absolutely continuous, invariant, probability measure (acip) if and only if the logarithm of the derivative is Lebesgue-integrable (see [14] for the recent exponential family analogue of this result and [34 for statistical properties of the maps considered in [3]). Thunberg, in [33, showed Benedicks-Carleson type results for unimodal families of maps with critical behaviour like $\exp \left(-|x|^{-\alpha}\right)$ for $\alpha<1 / 8$. He asked whether for $\alpha \geq 1$ no acip can exist.

For maps with non-flat critical points (that is, with critical behaviour like $|x|^{l}$ for some $l>1$ ) the log of the derivative is integrable. The maps considered by Thunberg have flat critical points, and the log of the derivative is integrable if and only if $\alpha<1$. Then by [3] no acip exists when both $\alpha \geq 1$ and the critical point is non-recurrent. Non-recurrence is historically a very important condition, but it is quite restrictive, see [32, 2]. 
For the maps considered in [3], Lebesgue measure is ergodic, so any acip necessarily has positive entropy, and thus positive Lyapunov exponent - see Ruelle's inequality below.

We drop the non-recurrence condition and the negative Schwarzian condition, extending the only if part of Benedicks and Misiurewicz's result to more general $C^{1+\epsilon}$ maps:

Theorem 1 Let $f: I \rightarrow I$ be a $C^{1+\epsilon}$ map of the compact interval I which is piecewise monotone. Suppose $f$ has an ergodic acip $\mu$ with positive Lyapunov exponent. Then the support of $\mu$ is a finite union of intervals on which

$$
\int \log |D f(x)| d x>-\infty
$$

where integration is with respect to Lebesgue measure.

Definition 2 If $A \subset \mathbb{R}$, we say a function $g: A \rightarrow \mathbb{R}$ is piecewise monotone if and only if there is a finite collection $\mathcal{Q}$ of pairwise disjoint intervals, whose union contains $A$, such that for each $Q \in \mathcal{Q}$, the restriction $g_{\mid Q}$ of $g$ to $Q$ is monotone (and not necessarily strictly monotone). We call such a $\mathcal{Q}$ a finite partition into intervals of monotonicity.

Since the derivative is bounded in the smooth setting, $\int \log |D f| d \mu \in[-\infty,+\infty)$, and in particular it exists. Ruelle's inequality for $C^{1}$ maps ([30] gives

$$
h_{\mu} \leq \max \left(0, \int \log |D f| d \mu\right)
$$

where $h_{\mu}$ denotes the entropy of $\mu$, so one can replace positive Lyapunov exponent by positive metric entropy in the hypotheses, if one so desires. Both our proof and that of [3] are based on showing that the density of the hypothetical acip is bounded away from zero. In the non-recurrent Misiurewicz setting, one then shows that the first return time to a sufficiently small critical neighbourhood is integrable if and only if (11) holds. On the other hand, for $C^{2}$ unimodal maps whose periodic points are all hyperbolic repelling, if the critical point is recurrent then the return time to a critical neighbourhood is always integrable. In particular, the techniques of [3] do not work in the recurrent general setting.

The following corollary responds to the question of Thunberg.

Corollary 3 Let $g_{b}$ be a map from the unimodal family

$$
x \mapsto-1+b\left(1-e^{-1-|x|^{-\alpha}}\right) .
$$

If $\alpha \geq 1$ then no ergodic acip with positive Lyapunov exponent exists.

Proof: Were such a measure to exist, its support would contain an interval, by Theorem 11 and so would necessarily contain the critical point at 0 . Then Theorem 1 would imply that $\log \left|D g_{b}(x)\right|$ is integrable with respect to Lebesgue measure. But $\log \left|D g_{b}(x)\right|=h(x)-|x|^{-\alpha}$, where $h$ is some function integrable with respect to Lebesgue measure. If $\alpha \geq 1$ then $|x|^{-\alpha}$ is not integrable. 


\subsubsection{On hypotheses and limitations}

For these results, ergodicity is not strictly necessary. As per [22, if one just supposes that $\mu$ is absolutely continuous and that $\mu$ almost every point has positive (pointwise) Lyapunov exponent, then one can use the ergodic decomposition theorem and continue similarly. In the interest of brevity and clarity we do not do this here.

The assumption that the acip has positive Lyapunov exponent is not wholly unnatural. If there exists a $\delta>0$ such that almost every point is contained in arbitrarily small intervals each mapped with universally bounded distortion by some iterate of $f$ onto some interval of length $\delta$, then it is not hard to show (with a density point argument) that any absolutely continuous measure actually has positive metric entropy, and that there are at most a finite number of such measures. Then, by Ruelle's inequality, the measure has positive Lyapunov exponent. Let us say that such maps have the large scale property.

In the non-flat setting, it is known that Lebesgue measure is ergodic 26, 4, and that any acip must have positive entropy. Outside the non-flat setting (and in particular for the unimodal family considered here), this is unknown and difficult. There may conceivably be maps with acips with negative or zero Lyapunov exponents. However, it should be possible to construct maps from the above unimodal family which have the large scale property, even if $\alpha \geq 1$, by controlling the rate of recurrence of the critical point. For such maps, Lebesgue measure would be conservative and ergodic, but no acip would exist.

While such maps probably exist, we do not believe that there is a positive measure set of parameters for which Lebesgue measure is conservative. We call a unimodal map hyperbolic if it has an attracting periodic orbit with a basin of attraction whose complement is (uniformly) hyperbolic repelling. For hyperbolic unimodal maps, Lebesgue almost every point lies in the basin of attraction, and Lebesgue measure is not conservative.

Conjecture 4 Consider the unimodal family

$$
g_{b}: x \mapsto-1+b\left(1-e^{-1-|x|^{-\alpha}}\right) .
$$

If $\alpha \geq 1$, then for Lebesgue almost every $b$, the map $g_{b}$ is hyperbolic.

In the theorem, we suppose that $f$ is piecewise monotone. We need this hypothesis to find a finite generating partition (see Proposition 29). Since the partition we find is finite, its entropy is finite. Without assuming finiteness of the number of turning points, it may be possible to find an infinite generating partition with finite entropy and other good properties, but we have not succeeded in doing so. Note that we do not exclude $f$ having an infinity of inflection points, or, a priori, $f$ having an interval of critical points, so it is a little more general than assuming that the set of critical points is finite. 


\subsection{Maps with unbounded derivative}

The second goal of the paper is to develop non-invertible Pesin theory for a class of maps with discontinuities and unbounded derivative. Map with unbounded derivative are of interest due to their links with the Lorenz map. See [25] and 24] for a discussion of this and [1] and [10] for existence results for absolutely continuous, invariant, probability measures. An early, general and very powerful result is by Rychlik [31].

In the following section we shall introduce a new class of maps called cusp maps. This class of maps will include all $C^{1+\epsilon}$ maps. For piecewise $C^{1+\epsilon}$ maps whose critical points verify a non-flatness condition, Pesin theory was studied by Ledrappier in 22. Given a measure with positive Lyapunov exponent, he showed existence of the unstable manifold in the natural extension, and several results which follow from it. The non-flatness hypothesis, used repeatedly in his proofs, means that we cannot use his results in the proof of Theorem 1

In Theorem 16, whose statement is overly technical for this introduction, we show existence of the unstable manifold for cusp maps. Even for $C^{1+\epsilon}$ maps our result is stronger than that of Ledrappier since we do not assume non-flatness of critical points. Moreover our proof is more direct. For $C^{1+\epsilon}$ maps one can also, with some work, deduce this result from [27; however the proof in that higher-dimensional setting is considerably more complex. This will allow us to prove Theorem 1 .

In [12, the author gave a $C^{r}$ version of Ledrappier's unstable manifold theorem and used it to prove $C^{r}$ conjugacy results. We shall also state a $C^{r}$ version here, but shall refer to 12 for the proof.

With unstable manifold in hand, we use regularly returning (or nice) intervals to give simple proofs of the dynamical volume lemma in Proposition 30 and of the existence of a Pesin partition in Proposition 31, compare [18] and 22.

Given a transformation $g$, we denote by $\mathcal{M}(g)$ the collection of ergodic $g$ invariant probability measures. If $f$ is a cusp map, $\mu \in \mathcal{M}(f)$ and $\chi_{\mu}:=$ $\int \log |D f| d \mu$ exists, then we call $\chi_{\mu}$ the Lyapunov exponent of $\mu$. For cusp maps, $\chi_{\mu}$ could be $+\infty$ or (which we do not exclude) $-\infty$, or it need not exist at all. In Section 11 there is an example of a cusp map with a measure of maximal entropy with Lyapunov exponent $+\infty$.

The paper culminates with the proof of the following result.

Theorem 5 Let $f$ be a cusp map which is piecewise monotone. Suppose $\mu$ is an ergodic, invariant, probability measure for $f$ with positive finite Lyapunov exponent $\chi_{\mu}$. The following conditions are equivalent:

1. $\mu$ is absolutely continuous with respect to Lebesgue measure;

2. $h_{\mu}=\chi_{\mu}$;

3. the density of $\mu$ with respect to Lebesgue measure is bounded from below by a positive constant on an open interval; 
4. $\mu$ is generated by a full expanding induced Markov map with integrable return time.

Proof: Follows immediately from Corollary 33 and Propositions 34 and 38 ,

We refer to Section 9 for the definition of a full expanding induced Markov map. The set on which the density is bounded away from zero is often large, see Lemma 35 and Theorem 36 .

Ledrappier 22 showed equivalence between 1 and 2 for $C^{1+\epsilon}$ maps with non-flat critical points. Bruin [5] showed equivalence of all four conditions in the case of unimodal maps with non-flat critical points and negative Schwarzian derivative. There is a recent related result in 8 for multimodal maps with nonflat critical points and negative Schwarzian derivative. Theorem 5 represents a substantial improvement.

We assume existence and finiteness of the Lyapunov exponent. As we have already stated, it need not exist. In Proposition 43, we construct benign examples of cusp maps with acips for which the Lyapunov exponent does not exist. We also show that cusp maps can have acips despite the presence of smooth parabolic fixed points, and that there are cusp maps for which the measure of maximal entropy has positive but infinite Lyapunov exponent.

The only reference measure we consider is Lebesgue measure. In the setting of holomorphic dynamics in 13, we consider more general conformal measures and invariant measures absolutely continuous with respect to them. It would be interesting to have similar results for conformal measures in the interval setting; we do not attempt this here.

\subsubsection{An alternative hypothesis: Positive entropy}

There is an alternative to assuming that the derivative should tend to 0 or $\pm \infty$ at the boundary of the domain of definition of the cusp map. We use the distortion bound and this assumption to guarantee that, when we pull back a small enough ball along a typical branch, we do not meet a discontinuity. An alternative approach is to assume positive entropy (as well as positive, finite, Lyapunov exponent).

Assuming positive entropy, we extend our results to a broader class of maps with a slightly more restrictive hypothesis on the measure. The Theorem 16 referred to in the following result says that along most inverse branches there is an interval that can be pulled back with good distortion control

Theorem 6 Let $f: \bigcup_{j=1}^{d} I_{j} \rightarrow I$ be a piecewise monotone map, a $C^{1}$ diffeomorphism on each subinterval $I_{j}$ of the compact interval $I$, which satisfies the conditions (2) and (3). Suppose $\mu \in \mathcal{M}(f)$ has positive, finite Lyapunov exponent and positive entropy.

Then the conclusions of Theorem 16 hold.

Since it was only in the proof of Theorem 16 that the assumption on the derivative at the boundary of the domain of definition was used, the other results also hold: 
Theorem 7 Let $f: \bigcup_{j=1}^{d} I_{j} \rightarrow I$ be a piecewise monotone map, a $C^{1}$ diffeomorphism on each $I_{j}$, satisfying the conditions (2) and (3). Suppose $\mu \in \mathcal{M}(f)$ has positive, finite Lyapunov exponent and positive entropy.

Then the conclusions of Theorem 5 and Propositions 29, 30 and 31 hold.

The assumption of positive entropy allows one to use the lift of the measure in the Hofbauer tower (or Markov extension) to study recurrence in the natural extension, and in particular to prove a result, Proposition 40, about existence of unstable fibres.

Proposition 40 is a topological (and metric) result. It does not give any information on distortion or lack thereof. For that we use positivity of the Lyapunov exponent.

For piecewise-monotone maps with bounded derivative, preceding results include [16, 17, 19, 21, 20.

\subsection{Structure of the paper}

The paper is structured as follows. In the next section we introduce the class of cusp maps. Then we prove some elementary distortion estimates. In Section 4 we define the natural extension and prove existence of the unstable manifold. In Section 5 we show the existence of useful regularly returning intervals. We then use these intervals to find a finite generating partition with good Markov properties and prove the dynamical volume lemma in Section 6. In Section 7 we show the existence of a Pesin partition of the natural extension. All results up to here are for an arbitrary ergodic invariant probability measure with positive finite Lyapunov exponent. In Section 8 we start the study of absolutely continuous measures and prove Theorem 1, In the following section we study induced Markov maps. Then in Section 10 we outline the proofs of Theorem 6 and Proposition 40. To finish up, in Section 11 we present some cusp maps conjugate to the Chebyshev map $x \mapsto 4 x(1-x)$ and look at some of their invariant measures.

\section{Definition of cusp maps}

Our goal is to develop the ergodic theory for piecewise smooth interval maps with singularities where the derivative, on at least one side, may tend to infinity.

For continuous maps with two smooth monotone branches, if the norm of the derivative tends to infinity as one approaches the turning point, the turning point is called a cusp. This leads us to introduce the following definitions. Throughout the paper, we shall denote by $I$ a non-degenerate compact interval.

Definition 8 A map $f: \bigcup_{j} I_{j} \rightarrow I$ is a basic cusp map if $\left(I_{j}\right)_{j}$ is a finite or countable collection of pairwise disjoint open subintervals of I such that $f$ is a $C^{1}$ diffeomorphism from each interval $\left(p_{j}, q_{j}\right):=I_{j}$ onto its image $f\left(I_{j}\right)$ and 
such that the following limits exist and equal either 0 or $\pm \infty$ :

$$
\lim _{x \rightarrow p_{j}^{+}} D f(x), \lim _{x \rightarrow q_{j}^{-}} D f(x) .
$$

Notice that $f$ is defined on a union of open intervals, a fact that is used repeatedly throughout the paper. For example, it implies that, given an interval $U, f^{n}$ is a diffeomorphism on each connected component of $f^{-n}(U)$, and each of these connected components is open. Choosing intervals to be open in the definition simplifies life later on, and from a measure-theoretical point of view, not having boundary points is unimportant.

We also note that the domain of definition of $f$ may have gaps, that is, the closure of the domain of definition need not be the entire interval $I$.

Let $A \subset \mathbb{R}$. Recall that a differentiable map $g: A \rightarrow \mathbb{R}$ is $C^{1+\epsilon}$ with constants $C$ and $\epsilon$ if, for all $x, x^{\prime}$ in $A$,

$$
\left|D g(x)-D g\left(x^{\prime}\right)\right| \leq C\left|x-x^{\prime}\right|^{\epsilon} .
$$

Definition 9 A basic cusp map $f: \bigcup_{j} I_{j} \rightarrow I$ is a cusp map (with constants $C, \epsilon)$ if there exist constants $C, \epsilon>0$ such that on each $I_{j}$,

- for all $x, x^{\prime}$ such that $\left|D f_{j}(x)\right|,\left|D f_{j}\left(x^{\prime}\right)\right| \leq 2$,

$$
\left|D f_{j}(x)-D f_{j}\left(x^{\prime}\right)\right| \leq C\left|x-x^{\prime}\right|^{\epsilon} ;
$$

- for all $x, x^{\prime}$ such that $\left|D f_{j}(x)\right|,\left|D f_{j}\left(x^{\prime}\right)\right| \geq 2^{-1}$,

$$
\left|\frac{1}{D f_{j}(x)}-\frac{1}{D f_{j}\left(x^{\prime}\right)}\right| \leq C\left|x-x^{\prime}\right|^{\epsilon} .
$$

One can check that poles of the form $x^{\alpha}$ where $0<\alpha<1$ (these are poles of root type) satisfy this latter relation. In figure 1 we present (the graphs of) some cusp maps.

Definition 10 A continuous map $g: I \rightarrow I$ which is $C^{1}$ on all but a countable set $S$ and for which $g_{\mid I \backslash(S \cup \text { Crit })}$ is a cusp map will also be called a cusp map. We use Crit to denote the set of critical points of $g_{\mid I \backslash S}$.

Since all our results will be for ergodic probability measures with positive finite Lyapunov exponent, the distinction between the continuous map and its restriction to $I \backslash(S \cup$ Crit) will be of no importance.

We are also interested in some analogue to $C^{r}$ maps. We want a condition which is satisfied by higher derivatives $D^{i} f$ of $f$ and which holds for all poles of root type. What appeared naturally and suffices to prove nice $C^{r}$ distortion properties for induced Markov maps is the following. 

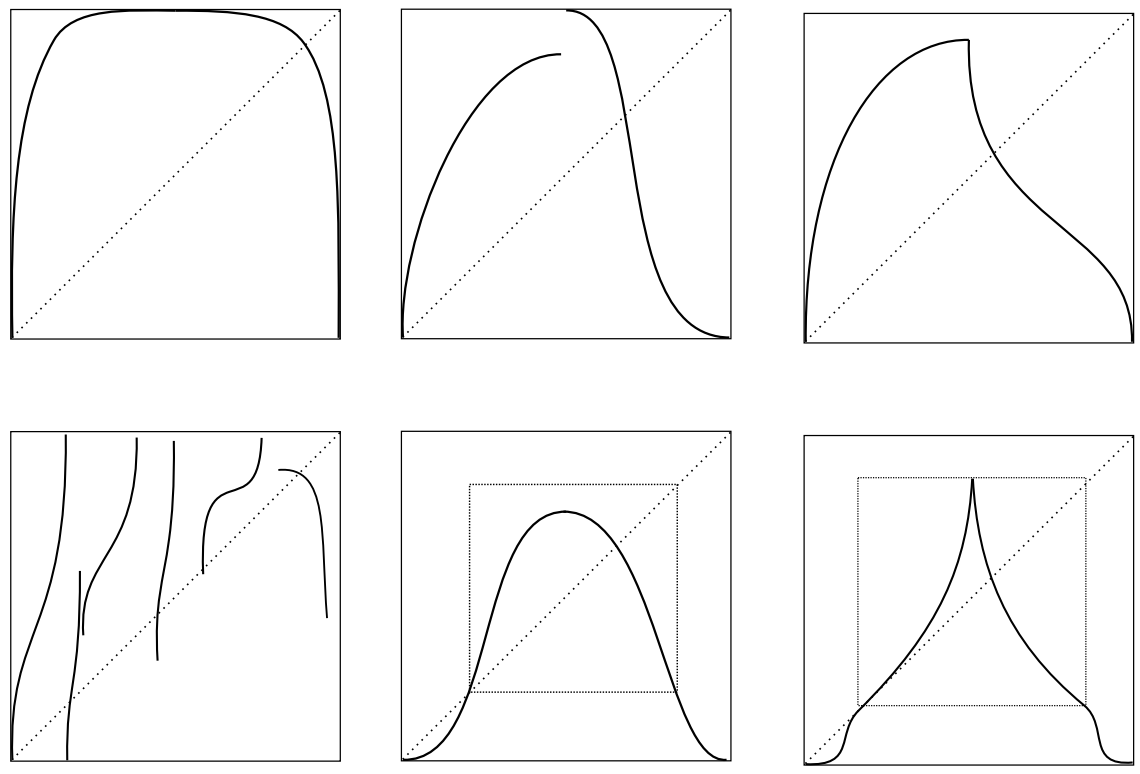

Figure 1: Some cusp maps.

Definition 11 Let $r \in\{2,3, \ldots\}$. A cusp map $f: \bigcup_{j} I_{j} \rightarrow I$ is a $C^{r}$ cusp map (with constants $p, C$ ) if there exist constants $C, p>1$ such that, for each $j$ one has:

- $f_{j}$ is $C^{r}$ on $I_{j}$;

- for all $x \in I_{j}$ such that $0<\left|D f_{j}(x)\right| \leq 2$, and for all $i$ such that $2 \leq i \leq r$, $\left|D^{i} f(x)\right|<C$;

- for all $x \in I_{j}$ with $\left|D f_{j}(x)\right| \geq 2$, and for all $i$ such that $2 \leq i \leq r$,

$$
\frac{\left|D^{i} f(x)\right|}{|D f(x)|^{p}}<C ;
$$

If $f$ is a $C^{r}$ cusp map for all integers $r>1$ then $f$ is a $C^{\infty}$ cusp map.

Definition 12 Let $f: I \rightarrow I$. A pole (at $p)$ is an interval $(p, p+\epsilon) \subset I$ such that $|\varepsilon|>0, f$ is $C^{1}$ on $(p, p+\epsilon)$ and

$$
\lim _{h \rightarrow 0^{+}}|D f(p+h \epsilon)|=\infty .
$$

\section{Distortion estimates}

We deduce some simple distortion estimates, culminating in Lemma 15] which roughly speaking says that the distortion is well-bounded on a ball of radius depending polynomially on the derivative. 
In the following two lemmas, one can think of $\phi$ as $D f$ if $D f$ is small, or as $\frac{1}{D f}$ if $D f$ is big.

Lemma 13 Let $c, C, \epsilon>0$ be positive constants. Let $\phi: A \rightarrow \mathbb{R}$ satisfy $\mid \phi(x)-$ $\phi\left(x^{\prime}\right)|\leq C| x-\left.x^{\prime}\right|^{\epsilon}$ for all $x, x^{\prime} \in A$.

Then, if $x, x^{\prime} \in A$ and $|\phi(x)|>c$,

$$
1-C c^{2} \frac{\left|x-x^{\prime}\right|^{\epsilon}}{c^{3}} \leq \frac{\phi\left(x^{\prime}\right)}{\phi(x)} \leq 1+C c^{2} \frac{\left|x-x^{\prime}\right|^{\epsilon}}{c^{3}} .
$$

Proof: We have $\left|\phi(x)-\phi\left(x^{\prime}\right)\right| \leq C\left|x-x^{\prime}\right|^{\epsilon}$ and $|\phi(x)|>c$. Thus

$$
\frac{\left|\phi(x)-\phi\left(x^{\prime}\right)\right|}{|\phi(x)|} \leq C \frac{\left|x-x^{\prime}\right|^{\epsilon}}{c}=C c^{2} \frac{\left|x-x^{\prime}\right|^{\epsilon}}{c^{3}} .
$$

One recovers (5) upon rewriting the left-hand side as $\left|1-\frac{\phi\left(x^{\prime}\right)}{\phi(x)}\right|$ and using the triangle inequalities.

Lemma 14 Let $C, \epsilon>0$ be constants. There exists a constant $c_{0}$, depending on $C, \epsilon$ and satisfying $0<c_{0}<\log 2$, with the following property:

If $\phi: A \rightarrow \mathbb{R}$ satisfies $\left|\phi(x)-\phi\left(x^{\prime}\right)\right| \leq C\left|x-x^{\prime}\right|^{\epsilon}$ for all $x, x^{\prime} \in A$, and if $c$ satisfies $0<c \leq c_{0}$, then for all $x, x^{\prime} \in A$ such that $\left|x-x^{\prime}\right|^{\epsilon}<c^{3}$ and $|\phi(x)|>c$,

$$
|\log | \phi(x)|-\log | \phi\left(x^{\prime}\right)|| \leq c \frac{\left|x-x^{\prime}\right|^{\epsilon}}{c^{3}}<c .
$$

Proof: There exists a constant $a_{0}$, with $0<a_{0}<\log 2$, such that $\log \left(1-\frac{a}{2}\right)>$ $-a$ for all $a$ satisfying $0<a<a_{0}$. Choose $c_{0}$ with $0<c_{0}<a_{0}$ and $C c_{0}<\frac{1}{2}$. Then by Lemma 13, for $c, x$ such that $0<c \leq c_{0}$ and $|\phi(x)|>c$,

$1-\frac{c}{2} \frac{\left|x-x^{\prime}\right|^{\epsilon}}{c^{3}} \leq 1-C c^{2} \frac{\left|x-x^{\prime}\right|^{\epsilon}}{c^{3}} \leq \frac{\phi\left(x^{\prime}\right)}{\phi(x)} \leq 1+C c^{2} \frac{\left|x-x^{\prime}\right|^{\epsilon}}{c^{3}} \leq 1+\frac{c}{2} \frac{\left|x-x^{\prime}\right|^{\epsilon}}{c^{3}}$.

Taking logs and using $c \leq c_{0}<a_{0}$,

$$
-c \frac{\left|x-x^{\prime}\right|^{\epsilon}}{c^{3}} \leq \log \left(\frac{\phi\left(x^{\prime}\right)}{\phi(x)}\right) \leq c \frac{\left|x-x^{\prime}\right|^{\epsilon}}{c^{3}},
$$

as required.

Lemma 15 Let $f: \bigcup_{j} I_{j} \rightarrow I$ be a cusp map with constants $C, \epsilon$.

There exists a constant $c_{0}>0$ such that if $c$ satisfies $0<c<c_{0}$ and $x, x^{\prime} \in \bigcup_{j} I_{j}$ satisfy $\left|x-x^{\prime}\right|^{\epsilon}<c^{3}$ and $c<|D f(x)|<c^{-1}$, then $x$ and $x^{\prime}$ are in the same component of the domain of definition of $f$ and

$$
|\log | D f(x)|-\log | D f\left(x^{\prime}\right)|| \leq c \frac{\left|x-x^{\prime}\right|^{\epsilon}}{c^{3}}<c .
$$


Proof: Let $c_{0}$ be given by Lemma 14, Fix $x \in \bigcup_{j} I_{j}$ and $c$ with $0<c<c_{0}$, such that $c<|D f(x)|<c^{-1}$. Let $A$ denote the connected component of

$$
\left\{x^{\prime} \in B\left(x, c^{(3 / \epsilon)}\right): 2^{-1}|D f(x)|<\left|D f\left(x^{\prime}\right)\right|<2|D f(x)|\right\}
$$

containing $x$; in particular $A$ is contained in a connected component of the domain of definition of $f$. If $|D f(x)| \leq 1$, set $\phi:=|D f|$, otherwise set $\phi:=$ $|D f|^{-1}$. By Lemma 14, we have

$$
|\log | D f(x)|-\log | D f\left(x^{\prime}\right)|| \leq c \frac{\left|x-x^{\prime}\right|^{\epsilon}}{c^{3}}<c<\log 2
$$

for all $x^{\prime} \in A$. It follows immediately that $A=B\left(x, c^{(3 / \epsilon)}\right)$.

\section{Unstable Manifold}

We define the natural extension as per [23. Let

$$
Y:=\left\{y=\left(y_{0} y_{1} y_{2} \ldots\right): f\left(y_{i+1}\right)=y_{i} \in I\right\} .
$$

Define $F^{-1}: Y \rightarrow Y$ by $F^{-1}\left(\left(y_{0} y_{1} \ldots\right)\right):=\left(y_{1} y_{2} \ldots\right)$. Then $F^{-1}$ is invertible with inverse $F: F^{-1}(Y) \rightarrow Y$. The projection $\Pi: Y \rightarrow I$ is defined by $\Pi: y=\left(y_{0} y_{1} \ldots\right) \mapsto y_{0}$. Then $\Pi \circ F=f \circ \Pi$. Given any measure $\mu \in \mathcal{M}(f)$ there exists a unique $F$-invariant measure $\bar{\mu}$ such that $\Pi_{*} \bar{\mu}=\mu$. Moreover $\bar{\mu} \in \mathcal{M}(F)$ and $\bar{\mu} \in \mathcal{M}\left(F^{-1}\right)$ (see [29]).

We call the triplet $(Y, F, \bar{\mu})$ the natural extension of $(f, \mu)$ (it is also called the Rohlin extension or the canonical extension).

Let us remark that invariant probability measures give no mass to the sets of points $x$ for which there is an $n>0$ such that $f^{n}(x)$ is not defined, nor do they give mass to the set of $x$ for which there exists an $n>0$ and no solution $x^{\prime}$ to $f^{n}\left(x^{\prime}\right)=x$. Thus, $F^{n}(y)$ is defined for all $n \in \mathbb{Z}$ for $\bar{\mu}$ almost every $y \in Y$.

Theorem 16 Let $f: \bigcup_{j} I_{j} \rightarrow I$ be a cusp map. Suppose $\mu \in \mathcal{M}(f)$ has positive finite Lyapunov exponent $\chi=\int \log |D f| d \mu>0$. Denote by $(Y, F, \bar{\mu})$ the natural extension of $(f, \mu)$.

Then there exists a measurable function $\alpha$ on $Y, 0<\alpha<1 / 2$ almost everywhere, such that for $\bar{\mu}$ almost every $y \in Y$ there exists a set $V_{y} \subset Y$ with the following properties:

- $y \in V_{y}$ and $\Pi V_{y}=B(\Pi y, \alpha(y))$;

- for each $n>0, f^{n}: \Pi F^{-n} V_{y} \rightarrow \Pi V_{y}$ is a diffeomorphism (in particular it is onto);

- for all $y^{\prime} \in V_{y}$

$$
\sum_{i=1}^{\infty}|\log | D f\left(\Pi F^{-i} y^{\prime}\right)|-\log | D f\left(\Pi F^{-i} y\right)||<\log 2 ;
$$


- for each $\eta>0$ there exists a measurable function $\rho$ on $Y, 0<\rho(y)<\infty$ almost everywhere, such that

$$
\rho(y)^{-1} e^{n(\chi-\eta)}<\left|D f^{n}\left(\Pi F^{-n} y\right)\right|<\rho(y) e^{n(\chi+\eta)} .
$$

In particular, $\left|\Pi F^{-n} V_{y}\right| \leq \rho(y) e^{-n(\chi-\eta)}$.

The above theorem is sufficient for the purposes of this paper. A stronger version is possible:

Theorem 17 Supplementarily to Theorem 16, there exists measurable $\gamma_{1}<\infty$ $\bar{\mu}$-almost everywhere such that for all $y^{\prime} \in V_{y}$,

$$
\sum_{i=1}^{\infty}|\log | D f\left(\Pi F^{-i} y^{\prime}\right)|-\log | D f\left(\Pi F^{-i} y\right)|| \leq \gamma_{1}(y)\left|\Pi y-\Pi y^{\prime}\right|^{\epsilon} .
$$

If $f$ is also a $C^{r}$ cusp map for some integer $r \geq 2$, then there exists a measurable function $\gamma_{r}$, with $\gamma_{r}(y)<\infty \bar{\mu}$-almost everywhere, such that for all $n$ and all $i=1,2, \ldots, r-1$,

$$
\frac{1}{\left|D f^{n}\left(\Pi f^{-n} y\right)\right|}\left|D^{i} \log \right| D f^{n}\left(\Pi f^{-n} y\right)|| \leq \gamma_{r}(y) .
$$

Proof: We refer to Proposition 2.11 of [12] for the proof. Our definition of $C^{r}$ cusp maps allows that proof to be applied directly. The full proof for cusp maps is also available in [11.

The rest of this section is devoted to the proof of Theorem 16 It will be broken up into several lemmas. The strategy is as follows. We have shown in Lemma 15 that we have a good distortion bound on

$$
B\left(x, \min \left(c_{0}^{3 / \epsilon},|D f(x)|^{ \pm 3 / \epsilon}\right)\right)
$$

for some constant $c_{0}$. Next we show that the derivative $\left|D f\left(\Pi F^{-n} y\right)\right|$ along backwards orbits is bounded from below by a sub-exponential sequence almost everywhere. This allows us to define a slowly-shrinking sequence of balls on which one has (slow-) exponentially good distortion. Positive Lyapunov exponent will then imply that the pullbacks of some small ball will always land inside the sequence of balls with exponentially good distortion bounds, so the total distortion will be summable.

Proof: We will need to swallow up some constants. Fix $\delta>0$ such that $\delta<\eta$, $(\chi-3 \delta)>3 \delta / \epsilon$. Subsequently fix $N>0$ large enough that, for all $n \geq N$, the following inequalities hold

$$
\begin{aligned}
2^{-1} \log 2+\sum_{m \geq N} e^{-m \delta} & <\log 2 \\
e^{-n \delta} & <c_{0} \\
2 e^{-n(\chi-\delta)} & <2^{-1} e^{-(n+1) \delta(1+3 / \epsilon)},
\end{aligned}
$$

where $c_{0}$ comes from Lemma 15 
Lemma 18 For $\bar{\mu}$ almost every $y$, there exists $n(y) \geq N$ such that for all $n \geq n(y)$,

$$
2 e^{-n \delta}<\left|D f\left(\Pi F^{-n} y\right)\right|<e^{n \delta} / 2
$$

and

$$
e^{n(\chi-\delta)} \leq\left|D f^{n}\left(\Pi F^{-n} y\right)\right| \leq e^{n(\chi+\delta)} .
$$

Proof: The first holds because the limit of $(1 / n) \log \left|D f^{n}\left(\Pi F^{-n} y\right)\right|$ exists for almost all $y$; the second because it equals $\chi$.

Lemma 19 Let $B_{n}:=B\left(\Pi F^{-n} y, 2^{-1} e^{-n 3 \delta / \epsilon}\right)$. For all $n \geq n(y), B_{n}$ is contained in the domain of definition of $f$ and, for all $x, x^{\prime} \in B_{n}$,

$$
|\log | D f(x)|-\log | D f\left(x^{\prime}\right)||<e^{-n \delta} .
$$

Proof: Follows from Lemmas 15, 18 ,

Lemma 20 For $n \geq n(y), f\left(B_{n+1}\right) \supset B\left(\Pi F^{-n} y, 2 e^{-n(\chi-\delta)}\right)$.

Proof: By the preceding lemmas, $|D f(x)|>e^{-(n+1) \delta}$ on $B_{n+1}$, so $f\left(B_{n+1}\right) \supset$ $B\left(\Pi F^{-n} y, 2^{-1} e^{-(n+1) 3 \delta / \epsilon} e^{-(n+1) \delta}\right)$. Then use (10).

Lemma 21 Suppose $n \geq n(y)$ and $V$ is an open ball containing $\Pi y$ with $|V|<1$ and suppose $V_{n}$ is such that $V_{n} \ni \Pi F^{-n} y$ and $f^{n}: V_{n} \rightarrow V$ is a diffeomorphism with distortion bounded by some $r$ with $0<r<\log 2$, i.e.,

$$
|\log | D f^{n}(x)|-\log | D f^{n}\left(x^{\prime}\right)||<r<\log 2
$$

for all $x, x^{\prime} \in V_{n}$. Then there exists $V_{n+1} \ni \Pi F^{-(n+1)} y$ such that the map $f^{n+1}: V_{n+1} \rightarrow V$ is a diffeomorphism with distortion bounded by

$$
r+e^{-(n+1) \delta} .
$$

Proof: We have that $\left|D f^{n}\left(\Pi F^{-n} y\right)\right|>e^{n(\chi-\delta)}$ so, by the distortion bound on $f^{n},\left|V_{n}\right|<2 e^{-n(\chi-\delta)}$ and $V_{n} \subset f\left(B_{n+1}\right)$. The result follows.

Now let $V$ be a sufficiently small ball centred on $\Pi y$ that there exists a set $V_{n(y)} \ni \Pi F^{-n(y)}(y)$ such that $f^{n(y)}: V_{n(y)} \rightarrow V$ is a diffeomorphism and for all $x, x^{\prime} \in V_{n(y)}$,

$$
\sum_{i=0}^{n(y)-1}|\log | D f\left(f^{i}(x)\right)|-\log | D f\left(f^{i}\left(x^{\prime}\right)\right)|| \leq(1 / 2) \log 2 .
$$

For $0 \leq n<n(y)$ define $V_{n}:=f^{n(y)-n}\left(V_{n(y)}\right)$. For $n>n(y)$, define $V_{n}$ inductively using Lemma 21. For any $n>0$, for any $x, x^{\prime} \in V_{n}$, we have

$$
\sum_{i=0}^{n-1}|\log | D f\left(f^{i}(x)\right)|-\log | D f\left(f^{i}\left(x^{\prime}\right)\right)|| \leq(1 / 2) \log 2+\sum_{j=n(y)}^{\infty} e^{-n \delta}<\log 2 .
$$

Define $V_{y}$ as the set of $y^{\prime} \in Y$ such that $\Pi F^{-n}\left(y^{\prime}\right) \in V_{n}$ for all $n \geq 0$. Let $\alpha(y)$ be the radius of $V$. Note that one can choose [ $n(y)$ minimal, so it is measurable, and then] $V$ [maximal] so that $\alpha$ is measurable. The existence of $\rho$ is easy, by ergodicity. This completes the proof of Theorem 16. 


\section{Regularly returning intervals}

The following lemma is simple and known. We include the proof for completeness and as an introduction to the arguments we will use later on, concerning points 'going to the large scale'.

Lemma 22 Let $f$ be a cusp map and suppose $\mu \in \mathcal{M}(f)$ has positive finite Lyapunov exponent $\chi_{\mu}$.

Inside any interval $U$ of positive measure, there are positive measure intervals $W \subset V$ and $n$ large such that $f^{n}$ maps $W$ diffeomorphically onto $V$ with $\left|D f_{\mid W}^{n}\right|>2$. The closure of the set of repelling periodic points has full $(\mu-)$ measure. The closure of the collection of inverse images (i.e. the closure of the backward orbit) of some periodic point has full measure.

Proof: Let $U$ be an open interval of positive measure. Let $(Y, F, \bar{\mu})$ be the natural extension for $(f, \mu)$. Modulo a set of $\bar{\mu}$-measure zero one can write

$$
\Pi^{-1} U=\bigcup_{k>0}\left\{y \in \Pi^{-1} U: \alpha(y)>\frac{1}{k}, \rho(y)<k\right\},
$$

where the functions come from Theorem[16, for $\eta<\chi_{\mu}$ a small positive constant. There is therefore a $k_{0}>2$ such that the set

$$
B:=\left\{y \in \Pi^{-1} U: \alpha(y)>\frac{1}{k_{0}}, \rho(y)<k_{0}\right\}
$$

is of positive $\bar{\mu}$ measure, i.e., $\bar{\mu}(B)>0$, and of course $\Pi(B) \subset U$. Then there is an open interval $V \subset U$ such that $|V|<k_{0}^{-1}$ and $\bar{\mu}\left(B \cap \Pi^{-1} V\right)>0$. Set $V_{B}:=B \cap \Pi^{-1} V$. By ergodicity, almost every point $y \in Y$ enters $V_{B}$ infinitely often, at times $n_{j}(y)$, say. Then $\Pi y$ is contained in intervals $W_{j}$ of size less than $k_{0} e^{-n_{j}(\chi-\eta)}$ mapped by $f^{n_{j}}$ with uniformly bounded distortion onto $V$. Taking $y \in \Pi^{-1} V$ and $n_{j}$ large, by the Intermediate Value Theorem there is a periodic point $p$ in $W_{j} \subset V \subset U$ and, by bounded distortion, it is repelling.

Similarly, almost every point in $I$ is contained in arbitrarily small intervals which get mapped onto $V$, so we conclude that the closure of the set of inverse images of $p$ has full measure.

Now we shall prove a general lemma which we were unable to find in the literature.

We say that a set $V$ is a one-sided neighbourhood of a point $p$ if it contains a small interval $(p, p+\varepsilon)$ for some non-zero real $\varepsilon$.

Lemma 23 Let $f: \bigcup_{j=1}^{n} U_{j} \rightarrow U$ be a piecewise continuous map, continuous on each (pairwise disjoint) open interval $U_{j}$, for which $f_{\mid U_{J}}$ extends to a continuous map on the compact interval $\overline{U_{j}}$ for each $j$.

Suppose $\mu$ is an ergodic, invariant, probability measure, and $W \subset f(W)$ is an interval of positive measure. Then there exists $k$ such that $\mu\left(f^{k}(W)\right)=1$. 
Proof: Let $Y$ denote the boundary set $\partial\left(\bigcup_{j} U_{j}\right)$ which is finite. Let $W_{j}=$ $\bigcup_{i=0}^{j} f^{i}(W)=f^{j}(W)$. Since $Y$ is finite, there is an $N$ such that for all $j>N$, $W_{j} \backslash W_{N}$ does not form a one-sided neighbourhood of any point in $Y$.

Suppose $j \geq N$ and $V$ is a connected component of $W_{j} \backslash Y$. Suppose there is some $k \geq 1$ such that $f^{k}(V) \cap W_{N} \neq \emptyset$ and let $l$ be the least such $k$. Then $f_{\mid V}^{l}$ is continuous.

For each connected component $V$ of $W_{N} \backslash Y$, let $n_{V}$ be the minimal $k \geq 1$ such that $f^{k}(V) \cap W_{N} \neq \emptyset$ if such a $k$ exists; otherwise set $n_{V}=0$. Let $M$ be the maximum of the $n_{V}$, noting that $W_{N}$ has a finite number of connected components. Let $X$ be a connected component of $W_{j}$ for some $j \geq M$. Suppose $X$ does not contain a connected component of $W_{M}$. Let $y \in X$ and let $l \geq 1$ be minimal such that $y \in f^{l}(V)$, where $V$ is some component of $W_{N} \backslash Y$. Then $l>M$. In particular, $n_{V}=0$, so $f^{i}(y) \notin W_{N}$ for all $i \geq 0$. This holds for each $y \in X$. Therefore $f^{i}(X) \cap W_{N}=\emptyset$ for all $i \geq 0$. By ergodicity, $X$ then has null measure.

Let $V_{1}, \ldots, V_{r}$ denote the connected components of $W_{M}$. For $j \geq M$ and $1 \leq k \leq r$, let $V_{k}^{j}$ denote the connected component of $W_{j}$ containing $V_{k}$. These are the only connected components of $W_{j}$ which may have positive measure.

Let $W_{\infty}=\bigcup_{j \geq 0} W_{j}$. We shall say that a point $x$ has the one-sided property if $W_{\infty}$ contains arbitrarily small, nested, one-sided neighbourhoods $J_{k}$ of the $x$, that each $J_{k}$ has positive measure, and that $J_{k} \not \subset W_{j}$ for any $j<\infty$ and that $\left|J_{k}\right| \rightarrow 0$.

Note that if the conclusion of the lemma does not hold then there exists a point $x$ which has the one-sided property. Suppose this is so. We must arrive at a contradiction.

Points with the one-sided property belong to the finite set $\bigcup_{k=1}^{r} \partial \bigcup_{j \geq M} V_{k}^{j}$. If $y$ is in the interior of $W_{\infty}$, then $f^{k}(y)$ does not have the one-sided property for any $k \geq 0$. Suppose $x$ has the one-sided property and let $J_{x}$ be a corresponding one-sided neighbourhood of $x$. Then there is a sequence $\left\{y_{n}\right\}$ for which $f\left(y_{n}\right) \in$ $J_{x}$ and converges to $x$ and for which each neighbourhood of each $y_{n}$ has positive measure. Replacing by a subsequence if necessary, we can assume the sequence $\left\{y_{n}\right\}$ is monotone. Let $y$ be its limit. Then $f\left(\left(y_{n}, y\right)\right)$ contains $\left(f\left(y_{n}\right), x\right) \subset J_{x}$. Since $x$ has the one-sided property, $\left(y_{n}, y\right) \not \subset W_{j}$ for any $j>0$, so $y$ has the one-sided property. Moreover, if $y_{n} \in U_{i}$ for all large $n$, then the continuous extension of $f$ to $\overline{U_{i}}$ maps $y$ to $x$.

It follows that (the finite collection of) points having the one-sided property are periodic, in the sense that if $x, J_{x}$ are as before and $J_{x}$ is sufficiently small then some iterate $f^{k}\left(J_{x}\right)$ is a small one-sided neighbourhood of $x$. We used here that on each $U_{i}$ the map $f$ extends continuously to the boundary.

The point $x$ is a fixed point of the continuous extension $g$ of $f_{\mid J_{x}}^{k}$ to the closure $\overline{J_{x}}$. If $g\left(J_{x}\right) \subset J_{x}$ for a sequence of arbitrarily small $J_{x}$ then $x$ must be a periodic orbit supporting the measure, by ergodicity. Suppose the measure is not supported on a periodic orbit (the lemma is trivial otherwise). Then $x$ is (one-sided, topologically-) repelling fixed point for $g$. For mass to accumulate near $x$ in $J_{x}$, there must be a point $y \neq x$ and arbitrarily small, positive measure, 
one-sided neighbourhoods $J_{y}$ of $y$ mapped by $f^{k}$ onto one-sided neighbourhoods of $x$ in $J_{x}$. But $y, J_{y}$ are not periodic in the above sense, so $y$ does not have the one-sided property. In particular, some sufficiently small $J_{y}$ is contained in some $W_{j}$. Then $W_{j+k} \supset f^{k}\left(J_{y}\right)$ contains some $J_{x}$, contradicting the one-sided property.

So far, our cusp maps may have an infinite (but countable) number of discontinuities. In the following definition and lemma, we wish to consider maps for which the number of serious discontinuities is finite.

Definition 24 We say a cusp map $f: \bigcup_{j} I_{j} \rightarrow I$ has a finite number of discontinuities if it extends to a continuous map $f_{*}$ on $\overline{\bigcup_{j} I_{j}} \backslash X$, where $X \subset$ $I \backslash \bigcup_{j} I_{j}$ is a finite set of points called points of discontinuity, and $X$ contains $\partial \widehat{\bigcup_{j} I_{j}}$.

Lemma 25 Let $f$ be a piecewise monotone cusp map with a finite number of discontinuities. Suppose $\mu \in \mathcal{M}(f)$ has a positive finite Lyapunov exponent.

Then given any interval $V$ of positive $\mu$-measure, there is a $j>0$ such that $\mu\left(\bigcup_{k=0}^{j} f^{k}(V)\right)=1$.

Proof: This follows immediately from Lemmas 23 and 22 .

Definition 26 ([15]) An open interval $U$ is regularly returning if $f^{n}(\partial U) \cap$ $U=\emptyset$ for all $n>0$. This is also called a nice interval in the literature.

For a cusp map $f$, if $A$ is a (necessarily open) connected component of $f^{-n}(U)$ and $B$ is a connected component of $f^{-m}(U)$ with $m \geq n$, it is easy to check that either $A \cap B=\emptyset$ or $B \subset A$, so inverse images of regularly returning intervals are either nested or disjoint. Indeed, suppose $x \in \partial A \cap B$. Then $f^{n}(x) \in \partial U$ (since $f$ may be discontinuous, one uses that $x \in B$ to know that $f^{n}$ is defined on a neighbourhood of $x$ ), but $f^{m}(x) \in U$, contradiction.

Lemma 27 Let $m$ be a non-atomic measure on $I$. Let $Z$ be the set of all $x \in I$ such that there exists an open interval $L$ with $m(L)=0$ and $x \in \partial L$. Then $m(Z)=0$.

Proof: Each open interval $L$ such that $m(L)=0$ is contained in a maximal such interval $V$. All such maximal intervals are pairwise disjoint. Thus there are at most a countable number of such maximal intervals $V$. But $m$ is non-atomic so $m(\partial V)=0$ and $Z$ is the countable union of sets of measure zero and thus of measure zero itself.

Proposition 28 Let $f$ be a cusp map and suppose $\mu \in \mathcal{M}(f)$ is non-atomic and has positive finite Lyapunov exponent.

Then $\mu$-almost every point is contained in arbitrarily small regularly returning open intervals, the boundaries of which are repelling periodic points. 
Proof: By Lemmas 27 and 22, almost every point $x$ is accumulated on both sides by repelling periodic points. Take one arbitrarily close periodic point, not in the orbit of $x$, on each side of $x$ and consider the partition defined by the orbits of these two points. The interior of each partition element, in particular the partition element which contains $x$, is regularly returning. The result follows, since the measure is non-atomic.

\section{Generating partition and the Dynamical Volume Lemma}

Given a map $f$ and a partition $\mathcal{P}$ we denote by $\mathcal{P}_{k}$ the partition $\bigvee_{i=0}^{k} f^{-i} \mathcal{P}$, and by $\mathcal{P}_{k}(x)$ the partition element containing the point $x$. Let $Y, \alpha, \rho$ be as per Theorem [16] and let $\gamma_{1}$ be as per Theorem 17. Recall that finite partitions into intervals of monotonicity are defined in Definition 2 .

Proposition 29 Let $f$ be a cusp map and suppose $\mu \in \mathcal{M}(f)$ is non-atomic and has positive finite Lyapunov exponent $\chi_{\mu}$. Suppose there exists a finite partition $\mathcal{Q}$ into intervals of monotonicity.

There exist a regularly returning interval $U$, constants $K, \varepsilon>0$, a finite partition $\mathcal{P}$ and a set $X$ of full measure with the following properties:

- $\bar{\mu}(A)>0$, where $A:=\left\{y \in Y: \Pi y \in U, \alpha(y) \geq|U|, \rho(y)<K, \gamma_{1}(y)<K\right.$ and $\left.\operatorname{dist}(\Pi y, \partial U)>2 \varepsilon|U|\right\} ;$

- $\mathcal{P}=\{U, I \backslash U\} \vee \mathcal{Q}$;

- $\mathcal{P}$ is generating;

- for each $x \in X$ there exists a strictly monotone increasing sequence $\left\{n_{j}\right\}$ such that

$$
f^{n_{j}}: \mathcal{P}_{n_{j}}(x) \rightarrow U
$$

is a diffeomorphism with distortion bounded by $\log 2$;

- $\operatorname{dist}\left(x, \partial \mathcal{P}_{n_{j}}(x)\right)>\varepsilon\left|\mathcal{P}_{n_{j}}(x)\right|$;

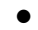

$$
\lim _{j \rightarrow \infty} \frac{j}{n_{j}}>0 \text { and } \lim _{j \rightarrow \infty} \frac{n_{j}}{n_{j+1}}=1 .
$$

Proof: There exists a $K>1$ such that

$$
B:=\left\{y \in Y: \alpha(y)>K^{-1} \text { and } \rho(y)<K\right\}
$$

has positive measure. By Proposition 28, one can cover a set of full measure by a countable collection of regularly returning intervals of diameter less than $K^{-1}$. Let $U$ be one such interval such that

$$
A_{0}:=B \cap \Pi^{-1} U
$$


has positive measure. Now $\mu(\partial U)=0$, so there exists a set $A \subset A_{0}$ verifying the first claimed property of the lemma for some $\varepsilon>0$.

By the Birkhoff Ergodic Theorem, almost every $y \in Y$ returns to $A$ at times $n_{j}(y)$ with $\lim _{j \rightarrow \infty} n_{j} / n_{j+1}=1$ and asymptotic frequency $\lim _{j \rightarrow \infty}\left(j / n_{j}\right)=$ $\bar{\mu}(A)$. Almost every point $x \in I$ is the projection of such a point $y$. Define $\mathcal{P}$ as above. By Theorem 16, $f^{n_{j}}$ maps $\mathcal{P}_{n_{j}}(x)$ diffeomorphically onto $U$; the partition elements shrink to the point $x$ as $j \rightarrow \infty$, so $\mathcal{P}$ is generating.

With this partition we can now give a very short proof of the following Dynamical Volume Lemma. For maps with "bounded $p$-variation", this was proven in 18. Maps with unbounded derivative do not have bounded $p$-variation. We denote by $\operatorname{HD}(\mu)$ the Hausdorff dimension of a measure $\mu$.

Proposition 30 Let $f$ be a cusp map and suppose $\mu \in \mathcal{M}(f)$ has positive finite Lyapunov exponent $\chi_{\mu}$. Suppose there exists a finite partition $\mathcal{Q}$ into intervals of monotonicity.

Then for $\mu$-almost every $x$,

$$
\lim _{r \rightarrow 0^{+}} \frac{\log \mu(B(x, r))}{\log r}=\frac{h_{\mu}}{\chi_{\mu}}
$$

in particular, $\operatorname{HD}(\mu)=h_{\mu} / \chi_{\mu}$.

Proof: The latter equality follows, by Frostman's Lemma, from the former, which we now prove. Note that if $\mu$ is atomic the proposition is trivial. We write $\chi$ for $\chi_{\mu}$. Let $(Y, F, \bar{\mu})$ be the natural extension. Let $\eta<\chi$ be a small positive constant. Let $\mathcal{P}=\left\{P_{0}=U, P_{1}, \ldots, P_{d}\right\}$ be a finite generating partition of $I, \varepsilon$ the constant and $X$ the set of full measure given by Proposition 29.

By ergodicity and the Shannon-McMillan-Breiman Theorem (28, p.39), there is a set $X^{\prime} \subset X$ of full measure such that the pointwise Lyapunov exponent $\lim _{n \rightarrow \infty}(1 / n) \log \left|D f^{n}(x)\right|=\chi$ and

$$
h_{\mu}=\lim _{n \rightarrow \infty} \frac{-1}{n} \log \mu\left(\mathcal{P}_{n}(x)\right)
$$

for all $x \in X^{\prime}$. Now fix $x \in X^{\prime}$ and let $n_{j}$ be given by Proposition 29,

Set $r_{j}:=\operatorname{dist}\left(x, \partial \mathcal{P}_{n_{j}}(x)\right)$ and $R_{j}=\left|\mathcal{P}_{n_{j}}(x)\right|$. We have (again by Proposition 29) that $r_{j} \geq \varepsilon R_{j}$. Continuing,

$$
\lim _{j \rightarrow \infty} \frac{1}{n_{j}} \log r_{j}=\lim _{j \rightarrow \infty} \frac{1}{n_{j}} \log R_{j}=-\chi
$$

and, since $\lim _{j \rightarrow \infty} \frac{n_{j}}{n_{j+1}}=1$,

$$
\lim _{j \rightarrow \infty} \frac{\log r_{j}}{\log r_{j+1}}=\lim _{j \rightarrow \infty} \frac{\log R_{j}}{\log R_{j+1}}=1 .
$$

Thus, if $r_{j} \geq r \geq r_{j+1}$,

$$
\frac{\log \mu(B(x, r))}{\log r} \geq \frac{\log \mu\left(\mathcal{P}_{n_{j}}(x)\right)}{\log r_{j+1}}=\frac{-1}{n_{j}} \log \mu\left(\mathcal{P}_{n_{j}}(x)\right) \frac{-n_{j}}{\log r_{j+1}}
$$


and the right-hand side tends to $\left(h_{\mu} / \chi\right)$ as $j \rightarrow \infty$.

If $R_{j} \geq r \geq R_{j+1}$,

$$
\frac{\log \mu(B(x, r))}{\log r} \leq \frac{\log \mu\left(\mathcal{P}_{n_{j+1}}(x)\right)}{\log R_{j}}=\frac{-1}{n_{j+1}} \log \mu\left(\mathcal{P}_{n_{j+1}}(x)\right) \frac{-n_{j+1}}{\log R_{j}}
$$

and the right-hand side tends to $\left(h_{\mu} / \chi\right)$ as $j \rightarrow \infty$.

As $r \rightarrow 0$ one has $j \rightarrow \infty$ so we conclude that

$$
\lim _{r \rightarrow 0} \frac{\log \mu(B(x, r))}{\log r}=\frac{h_{\mu}}{\chi}
$$

as required.

\section{Existence of Pesin partition}

An analogous result to the following was proven in [22] in a more restrictive setting, but the same proof works. However it is unnecessarily complicated. We provide a short proof of the existence of Pesin's partition, taking advantage of the properties of regularly returning intervals.

Proposition 31 Let $f$ be a piecewise-monotone cusp map. Suppose $\mu \in \mathcal{M}(f)$ has positive finite Lyapunov exponent and denote the natural extension $(Y, F, \bar{\mu})$. Let $\xi$ be the measurable partition of $Y$ defined by

$$
\xi=\bigvee_{i=0}^{\infty} F^{i}\left(\Pi^{-1} \mathcal{P}\right)
$$

where $\mathcal{P}$ is a partition given by Proposition 29. Then $\xi$ has the following properties:

1. the partition $\xi$ is increasing by $F, F^{-1} \xi>\xi$, and generates;

2. entropy of $\mu$ is given by $h(\mu)=H\left(F^{-1} \xi / \xi\right)$;

3. for $\bar{\mu}$ almost every point $y$, for all $k \geq 0$, $\Pi$ maps $F^{-k}(\xi(y))$ injectively into an interval of monotonicity of $f$, where $\xi(y)$ denotes the element of $\xi$ containing $y$;

4. for $\bar{\mu}$ almost every point $y, 0<\int_{\xi(y)} \Delta\left(y, y^{\prime}\right) d y^{\prime}<\infty$, where the integration is with respect to the natural Lebesgue measure (i.e. the pullback of Lebesgue measure by $\left.\Pi_{\mid \xi(y)}\right)$ on each element of $\xi$ and

$$
\Delta\left(y, y^{\prime}\right)=\lim _{n \rightarrow \infty} \frac{D f^{n}\left(\Pi F^{-n} y\right)}{D f^{n}\left(\Pi F^{-n} y^{\prime}\right)} ;
$$

5. let $U$ and $A$ be given by Proposition 29, so $\bar{\mu}(A)>0$ : for all $y \in A$, $\Pi \xi(y)=U$. 
Proof: Since $\mathcal{P}$ is finite and generating, $h(f, \mathcal{P})=h_{\mu}$. Let $\zeta$ be the partition of $Y$ given by $\zeta:=\left\{\Pi^{-1} P: P \in \mathcal{P}\right\}$ and let $\xi:=\bigvee_{i=0}^{\infty} F^{i} \zeta$. Then $\zeta$ is a finite, generating partition of $Y$, and

$$
h_{\bar{\mu}}=h(F, \zeta)=H\left(\zeta \mid \bigvee_{i>0} F^{i} \zeta\right)=H\left(F^{-1} \xi \mid \xi\right)=h(f, \mathcal{P})=h_{\mu}
$$

That $\Pi$ maps $F^{-k} \xi(y)$ injectively into an interval of monotonicity holds since $\mathcal{P}$ is a refinement of the partition into intervals of monotonicity.

For $y \in A$, that $\Pi \xi(y)=U$ follows from the regularly returning property. For almost every $y \in Y$, there is an $n \geq 0$ such that $F^{n} y \in A$. Then $\xi(y) \supset$ $F^{-n}\left(\xi\left(F^{n} y\right)\right)$, so $\Pi \xi(y)$ contains an open interval.

It remains to show that the integral is positive and finite, which follows as per [22: For clarity, let us write $\left[F^{-k} \xi\right]$ instead of $F^{-k} \xi$. For all $k \geq 0$, one has $\xi(y)=F^{k}\left(\left[F^{-k} \xi\right]\left(F^{-k} y\right)\right)$, so the projection of the partition element $\left[F^{-k} \xi\right]\left(F^{-k} y\right)$ contains an open interval for almost every $y$. For almost every $y,\left|D f\left(\Pi F^{-i} y\right)\right|$ is positive and finite for all $i$, and by ergodicity there exists a $k \geq 0$ such that $F^{-k} y \in A$. One has

$$
\begin{gathered}
\int_{\xi(y)} \Delta\left(y, y^{\prime}\right) d y^{\prime}=\int_{F^{k}\left(\left[F^{-k} \xi\right]\left(F^{-k} y\right)\right)} \Delta\left(F^{-k} y, F^{-k} y^{\prime}\right) \prod_{i=1}^{k} \frac{D f\left(\Pi F^{-i} y\right)}{D f\left(\Pi F^{-i} y^{\prime}\right)} d y^{\prime} \\
=\prod_{i=1}^{k}\left|D f\left(\Pi F^{-i} y\right)\right| \int_{\left[F^{-k} \xi\right]\left(F^{-k} y\right)} \Delta\left(F^{-k} y, y^{\prime}\right) d y^{\prime} .
\end{gathered}
$$

By the distortion bound of Proposition 29, the last integrand is bounded inside $\left(2^{-1}, 2\right)$ since $\left[F^{-k} \xi\right]\left(F^{-k} y\right) \subset \xi\left(F^{-k} y\right)$ and $F^{-k} y \in A$. Thus the integrals are positive and finite, completing the proof.

\section{Absolutely continuous measures}

The Rohlin decomposition $p(y, \cdot)$ for the measure $\bar{\mu}$ with respect to the partition $\xi$ is a conditional probability measure on each partition element of $Y$ such that, for any measurable set $B \subset Y$ one has

$$
\bar{\mu}(B)=\int_{Y} p(y, B) d \bar{\mu}=\int_{Y} p(y, B \cap \xi(y)) d \bar{\mu} .
$$

By Proposition 31, if $n>0$,

$$
n h_{\mu}=H\left(F^{-n} \xi / \xi\right)=-\int \log p\left(y,\left[F^{-n} \xi\right](y)\right) d \bar{\mu} .
$$

Proposition 32 Let $f$ be a piecewise-monotone cusp map. Suppose $\mu \in \mathcal{M}(f)$ has Lyapunov exponent $\chi_{\mu}$ and entropy $h_{\mu}$ satisfying $0<\chi_{\mu}=h_{\mu}<\infty$. Let $(Y, F, \bar{\mu})$ be the natural extension. 
Then the Rohlin decomposition for the measure $\bar{\mu}$ with respect to the partition $\xi$ of Proposition 31 is given by $q(y, B)$, for $y$ in $Y$ and $B$ a measurable subset, where

$$
q(y, B):=\frac{\int_{B \cap \xi(y)} \Delta\left(y, y^{\prime}\right) d y^{\prime}}{\int_{\xi(y)} \Delta\left(y, y^{\prime}\right) d y^{\prime}} .
$$

Proof: The proof carries over from [22], proposition 3.6, without modification.

Corollary 33 Let $f$ be a piecewise-monotone cusp map. Suppose $\mu \in \mathcal{M}(f)$ has positive finite Lyapunov exponent $\chi_{\mu}$.

If $h_{\mu}=\chi_{\mu}$, or equivalently if $\operatorname{HD}(\mu)=1$, then $\mu$ is absolutely continuous.

Proof: Let $B$ be a subset of zero Lebesgue measure. Then $q(y, B)=0$ for all $y$, so $\bar{\mu}\left(\Pi^{-1} B\right)=0=\mu(B)$.

Proposition 34 Let $f$ be a piecewise-monotone cusp map. Suppose $\mu \in \mathcal{M}(f)$ has positive finite Lyapunov exponent.

If $\mu$ is absolutely continuous with respect to Lebesgue measure then there exist $\nu>0$ and an open interval such that the density of $\mu$ is bounded from below by some constant $\nu>0$ Lebesgue almost everywhere on the interval.

Proof: Let $\xi, A, U$ be given by Proposition 31, For all $y \in A, \Pi \xi(y)=U$ and $2^{-1} \leq \Delta\left(y, y^{\prime}\right) \leq 2$ if $y^{\prime} \in \xi(y)$. Thus, for $y \in A$, the density of the Rohlin decomposition on the partition element $\xi(y)$ containing $y$ is bounded inside $\left[4^{-1}|U|^{-1}, 4|U|^{-1}\right]$. Let $A^{\prime}:=\bigcup_{y \in A} \xi(y)$. If we set $\bar{\mu}_{A}:=\bar{\mu}_{\mid A^{\prime}}$, then $\Pi_{*} \bar{\mu}_{A}$ has support $U$ and the density of $\Pi_{*} \bar{\mu}_{A}$ is bounded inside

$$
\left[4^{-1}|U|^{-1} \bar{\mu}\left(A^{\prime}\right), 4|U|^{-1} \bar{\mu}\left(A^{\prime}\right)\right] .
$$

Since $\bar{\mu}_{A}$ is a restriction of $\bar{\mu}$, the density of $\Pi_{*} \bar{\mu}_{A}$ is less than that of $\mu$ almost everywhere. In particular, the density of $\mu$ on $U$ is bounded from below by $\nu:=4^{-1}|U|^{-1} \bar{\mu}\left(A^{\prime}\right)>0$ as required.

Lemma 35 Let $g$ be a piecewise $C^{1}$ map with an absolutely continuous invariant probability measure with density $\rho$. Then

$$
\rho(x)=\sum_{w \in g^{-1} x} \frac{1}{|D g(w)|} \rho(w) .
$$

In particular, $\rho(g(x)) \geq \frac{1}{|D g(x)|} \rho(x)$.

Proof: This is just the change of variables formula.

Theorem 36 Let $f: I \rightarrow I$ be a piecewise-monotone cusp map with only a finite number of discontinuities. Suppose $\mu \in \mathcal{M}(f)$ has positive finite Lyapunov exponent and that $\mu$ is absolutely continuous with respect to Lebesgue measure. 
Then the support of $\mu$ is a finite union of intervals $X$ on which $\mu$ is equivalent to Lebesgue.

Moreover, on every compact subset of $X$ disjoint from the forward orbit of poles of $f$ the density is bounded away from 0 . In particular, if $f$ has no poles the density is bounded away from 0 on $X$.

Proof: Let $U$ be the interval given by Proposition 34 on which the density is bounded away from 0 . By Lemma 25, there exists a $j>0$ such that the closure $X$ of $\bigcup_{k=0}^{j} f^{k} U$ contains the support of $\mu$, and thus equals the support of $\mu$. The result then follows from Lemma 35.

Proof of Theorem [1: We can apply Theorem 36 since $f$ extends to be a continuous cusp map on some larger interval. The lower bound on the density implies that anything integrable with respect to $\mu$ is integrable with respect to Lebesgue measure on the support of $\mu$.

\section{Induced Markov maps}

When trying to prove the existence of absolutely continuous invariant probability measures, a standard and fruitful technique is to show the existence of an expanding induced map whose domain has full measure in its range. One can spread the absolutely-continuous, invariant, probability measure for the induced map to get an absolutely-continuous, invariant measure for the original map. If the return time for such a Markov map is integrable with respect to Lebesgue measure, then the resultant measure is finite and can be normalised to give a probability measure.

A natural question is whether for all acips the measure can be produced from such an induced map. Henk Bruin [5] has shown that this is the case for unimodal maps with negative Schwarzian derivative and non-flat critical points (this has now been extended to multimodal maps with negative Schwarzian derivative and non-flat critical points [8]). We prove a stronger result, dropping the condition on non-flatness of critical points, admitting poles and weakening the condition on the number of singularities.

Definition 37 Suppose $I^{\prime} \subset I$ and $f: I^{\prime} \rightarrow I$, where $I$ is an interval. Let $\left\{U_{i}\right\}$ be a finite or countable collection of disjoint open subintervals of an open interval $U \subset I^{\prime}$. We call a map $\phi: \bigcup_{i} U_{i} \rightarrow U$ an expanding induced Markov map if

- $\phi$ restricted to each $U_{i}$ is a diffeomorphism onto $U$;

- $|D \phi| \geq \lambda>1$ for some constant $\lambda$;

- there exist constants $C, \epsilon>0$ such that for each $i$, for all $x, x^{\prime} \in U_{i}$,

$$
\left|D \phi(x)-D \phi\left(x^{\prime}\right)\right| \leq C\left|\phi(x)-\phi\left(x^{\prime}\right)\right|^{\epsilon} ;
$$


- there exists $\left\{n_{i}\right\}$ such that $\phi_{\mid U_{i}}=f_{\mid U_{i}}^{n_{i}}$.

If moreover $U \backslash \bigcup_{i} U_{i}$ has zero Lebesgue measure, then we call $\phi$ full.

Let $n(x):=n_{i}$ if $x \in U_{i}$. If $\phi$ is full and

$$
\int_{U} n(x) d x=\sum_{i} n_{i}\left|U_{i}\right|<\infty
$$

then we say $\phi$ has integrable return time.

It follows easily from Proposition 29 that if $f$ is a cusp map and $\mu \in \mathcal{M}(f)$ has positive finite Lyapunov exponent, then there exists an expanding induced Markov map such that $\mu\left(\bigcap_{j \geq 0} \phi^{-j}(U)\right)=\mu(U)>0$ (note that holding of the Hölder condition comes from Theorem 17 via the $\gamma_{1}$ of Proposition 29). We want to show more than this in the case that $\mu$ is absolutely continuous.

The Folklore Theorem (see e.g. 9]) implies that if $\phi$ is a full expanding induced Markov map then $\phi$ has a unique absolutely continuous invariant probability measure, $\nu$ say, whose density is bounded away from zero and infinity on $U$.

If $\phi$ has integrable return time then, as is well known,

$$
\sum_{i} \sum_{j=0}^{n_{i}-1} f_{*}^{j} \nu_{\mid U_{i}}
$$

is a finite, ergodic, absolutely-continuous, invariant measure for $f$ which, when normalised, is an ergodic acip $\mu$ for $f$. We say $\mu$ is generated by $\phi$.

Proposition 38 Let $f$ be a piecewise-monotone cusp map. Suppose $\mu \in \mathcal{M}(f)$ has positive finite Lyapunov exponent and that $\mu$ is absolutely continuous with respect to Lebesgue measure.

Then $\mu$ is generated by a full expanding induced Markov map for $f$.

Proof: Let $A^{\prime}, U$ be defined as per the proof of Proposition 34. For $y \in Y$ let $r_{1}(y):=\inf \left\{n \geq 1: F^{n} y \in A^{\prime}\right\}$. Inductively define $r_{k+1}(y):=r_{k}(y)+r\left(F^{r_{k}(y)} y\right)$ for $k \geq 1$ and set $n_{k}(x):=\min \left\{r_{k}(y): \Pi(y)=x\right\}$. These are defined on sets of full measure, in particular for Lebesgue almost every $x \in U$. Recall we showed that the density of $\Pi_{*} \bar{\mu}_{\mid A^{\prime}}$ is bounded from below on $U$ by some $\nu>0$.

By an easy generalisation of Kac's Lemma, $\int_{A^{\prime}} r_{k}(y) d \bar{\mu}=k$ for each $k \geq 1$. Then

$$
k \geq \int_{U} n_{k}(x) d \Pi_{*} \bar{\mu}_{\mid A^{\prime}} \geq \nu^{-1} \int_{U} n_{k}(x) d x .
$$

In particular $\int_{U} n_{k}(x) d x<\infty$.

Recall that for $y \in A^{\prime}, \Pi \xi(y)=U$, and for $y \in A, \rho(y)<K$, so by Theorem 16. $\left|D f^{n}\right|>(2 K)^{-1} e^{n(\chi-\eta)}$ on $\Pi F^{-n} \xi(y)$. Choose $N$ such that $(2 K)^{-1} e^{N(\chi-\eta)}>2$. 
Let $D$ denote the set of $x \in U$ such that $n_{N}(x)$ is defined. Since $n_{N}(x)$ is defined almost everywhere, the Lebesgue measure of $U \backslash D$ is zero. For each $x \in D$ there exists $y \in \Pi^{-1}(x)$ such that $F^{n_{N}(x)} y \in A^{\prime}$. Set

$$
U_{x}:=\Pi F^{-r_{N}(y)} \xi\left(F^{r_{N}(y)}\right),
$$

and note that since $\xi\left(y^{\prime}\right)=\xi(y)$ for all $y^{\prime} \in \xi(y)$ and since $U$ is regularly returning, $U_{x}$ is a connected component of $D$. in particular, $n_{N}\left(x^{\prime}\right)$ is defined and constant on $U_{x}$. Let $\left\{U_{i}\right\}$ be the collection of connected components of $D$ and $m_{i}:=n_{N}(x)$ for some $x \in U_{i}$. Then define $\phi: \bigcup U_{i} \rightarrow U$ by $\phi_{\mid U_{i}}:=f^{m_{i}}$. This map is a full expanding induced Markov map.

Thus there is an ergodic invariant absolutely continuous invariant probability measure $\mu^{\prime}$ generated by $\phi$. The support of $\mu^{\prime}$ coincides with that of $\mu$ and both have positive density so by ergodicity they are equal and $\mu$ is generated by $\phi$.

Theorem 39 Let $f$ be a piecewise-monotone $C^{1+\epsilon}$ map.

Then $f$ has an ergodic, absolutely continuous, invariant, probability measure with positive finite Lyapunov exponent if and only if there exists a full expanding induced Markov map with integrable return time.

Proof: One direction is given by Proposition 38. On the other hand, if there exists an induced Markov map with integrable return time, the measure generated by it will be an ergodic, absolutely continuous, invariant probability measure. The entropy of the measure is positive because it is non-invertible almost everywhere on the range of the Markov map. Then Ruelle's Inequality implies that the Lyapunov exponent is positive, and it is finite because the derivative is bounded.

Remark: There are induced Markov maps with integrable return time for cusp maps such that the generated measure has non-integrable Lyapunov exponent, see the section after next.

\section{From Markov extension to natural extension}

This section builds on the work of Hofbauer ([16]) and Keller ([20]) and looks at the relation between their Markov extension or Hofbauer tower and the natural extension.

Consider a piecewise-monotone map $f: \bigcup_{j=1}^{d} I_{j} \rightarrow I$ of the interval $I$ defined on a collection of open intervals $I_{j}$ and an invariant, ergodic, probability measure $\mu$. As is well known, Hofbauer showed that if the entropy $h_{\mu}>0$, then $\mu$ lifts to an ergodic, conservative, $\widehat{f}$-invariant probability measure $\widehat{\mu}$ for the corresponding Markov extension $\widehat{f}: \widehat{\bigcup}_{j=1}^{d} I_{j} \rightarrow \widehat{I}$.

Bruin and Todd use this in the following way: There is some interval $\widehat{J}$ at some level in the tower which has positive measure. By conservativity, the domain of the first return map $\widehat{\phi}$ to $\widehat{J}$ has full measure. The topological structure 
of the tower implies that, when chosen appropriately, $\widehat{\phi}$ is a homeomorphism from each connected component of its domain onto $\widehat{J}$. This then also holds for all iterates of $\widehat{\phi}$. By conservativity, $\bigcap_{n \geq 0} \widehat{\phi}^{-n}(\widehat{J})$ has the same measure as $\widehat{J}$. See section 3 , and in particular subsection 3.2, of 8 for details.

We can look at the natural extension of the Markov extension. Since it projects onto the Markov extension which then projects onto the original system, it is none other than the natural extension $(Y, F, \bar{\mu})$ of the original system. Let $\widehat{\pi}$ denote the projection from $Y$ to $\widehat{I}$, and, as usual, $\Pi$ the projection from $Y$ to $I$.

Let $A \subset Y$ denote $\widehat{\pi}^{-1}(\widehat{J})$. Then $A$ has positive measure. Let $\Phi: A \rightarrow$ $A$ denote the first return map to $A$ and set $A_{*}:=\bigcap_{j \geq 0} \Phi^{-j}(A)$; again by conservativity, $A_{*}$ has positive measure. The first return map $\Phi$ projects down to the map $\widehat{\phi}$ on $\widehat{J}$, that is, $\widehat{\pi} \circ \Phi=\widehat{\phi} \circ \widehat{\pi}$ since $\widehat{\phi}$ is a first return map [note that the projection down to the original system in general is not a first return map].

Let $y \in A_{*}$ and $y_{j}=\Phi^{-j} y$. Then $\widehat{\phi}^{j}$ maps a neighbourhood $\widehat{W}_{j} \subset \widehat{J}$ of $\widehat{\pi}\left(y_{j}\right)$ homeomorphically onto $\widehat{J}$, so there is some $k_{j}$ and a corresponding neighbourhood $W_{j}$ of $\Pi y_{j} \in I$ mapped by $f^{k_{j}}$ homeomorphically onto $J$, the projection of $\widehat{J}$, and $f^{k_{j}} \circ \Pi=\Pi \circ \Phi^{j}$ on $\Pi^{-1} W_{j}$.

Then for each $x$ in $J$, there is a corresponding point $y^{x} \in \Pi^{-1} x$ such that for all $j \geq 0, \Pi \Phi^{-j}\left(y^{x}\right) \in W_{j}$. In particular, $\Pi \Phi^{-j}\left(\bigcup_{x \in J} y^{x}\right)=W_{j}$. It follows that for each $k \geq 0, \Pi F^{-k}\left(\bigcup_{x \in J} y^{x}\right)$ is an interval mapped homeomorphically by $f^{k}$ onto $J$.

We remark that almost every point $y$ lands in $A_{*}$ at some point. Thus the following holds.

Proposition 40 Suppose $f$ is a piecewise-monotone, piecewise-continuous map and $\mu$ is an ergodic, invariant, probability measure with positive entropy $\mu$. There is a measurable function $\theta$ on $Y$ such that for almost every $y$, there exists a set $V_{y} \subset Y$ with the following properties:

- $y \in V_{y}, \theta(y)>0$ and $\Pi V_{y}=B(\Pi y, \theta(y))$;

- for each $m>0, f^{m}: \Pi F^{-m} V_{y} \rightarrow \Pi V_{y}$ is a homeomorphism (in particular it is onto).

Now, the collection $\mathcal{Q}:=\left\{I_{1}, \ldots, I_{d}\right\}$ defines a partition of the domain of $f$ and $f$ is a homeomorphism on each $I_{j}$ (onto its image). If $f^{n}$ is a homeomorphism from some interval $W$ onto its image, then $W$ is entirely contained in one element of the partition $\mathcal{Q}$. Denote by $\mathcal{Q}(x)$ the partition element containing the point $x$.

By Proposition 40, for almost every $y$ there is a set $V_{y} \ni y$ for which $\Pi F^{-n}\left(V_{y}\right)$ is mapped homeomorphically onto the open interval $\Pi V_{y}$ by $f^{n}$. Thus $\Pi F^{-n}\left(V_{y}\right)$ is entirely contained in some element of $\mathcal{Q}$.

We now provide the remaining arguments needed to show Theorem 16 Lemma 13 almost holds: one must consider the intervals $I_{j}$ one at a time. Lemma 16 holds unchanged. Lemma 17 is modified slightly taking into account Lemma 13: 
Lemma 41 Let $B_{n}:=B\left(\Pi F^{-n} y, 2^{-1} e^{-n 3 \delta / \epsilon}\right)$. For all $n \geq n(y)$, for all $x, x^{\prime} \in$ $B_{n} \cap \mathcal{Q}\left(\Pi F^{-n}(y)\right)$,

$$
|\log | D f(x)|-\log | D f\left(x^{\prime}\right)||<e^{-n \delta} .
$$

Lemma 18 becomes:

Lemma 42 For $n \geq n(y), f\left(B_{n+1} \cap \mathcal{Q}\left(\Pi F^{-(n+1)} y\right) \supset B\left(\Pi F^{-n} y, 2 e^{-n(\chi-\delta)}\right) \cap\right.$ $f\left(\mathcal{Q}\left(\Pi F^{-(n+1)} y\right)\right)$.

Lemma 19 holds under the additional assumption that $V_{n} \subset f\left(\mathcal{Q}\left(\Pi F^{-(n+1)}\right)\right)$. This holds if $V$ is a subset of $\Pi V_{y}$, where $V_{y}$ comes from Proposition 40, The remainder of the proof follows through.

\section{Conjugacies with the Chebyshev map}

The Chebyshev quadratic map $x \mapsto 4 x(1-x)$ is a very special example as it is smoothly conjugate on the interior of $[0,1]$ to a piecewise linear map, namely the full tent map $T:[0,1] \rightarrow[0,1]:$

$$
T(x)= \begin{cases}2 x & \text { if } 0 \leq x \leq \frac{1}{2} \\ 2-2 x & \text { if } \frac{1}{2}<x \leq 1\end{cases}
$$

We shall use it to construct some examples of cusp maps. In Theorem 1 and Corollary 3 , we showed that for $C^{1+\epsilon}$ maps there are no absolutely continuous invariant measures with positive Lyapunov exponent if the critical points are too flat. Here we shall find unimodal maps with acips with positive entropy and very flat critical points. This is strange, but a contradiction is avoided because these maps have singularities at the boundary and the logarithm of the derivative is non-integrable, so the Lyapunov exponent of the acip does not exist.

Topological Chebyshev maps will also provide examples of maps whose measures of maximal entropy have infinite Lyapunov exponent.

Proposition 43 Let TC denote the class of cusp maps or restrictions of cusp maps which are topologically conjugate by a conjugacy $h$ to the full tent map $T$. Let $T C^{\infty}$ denote the subclass of such maps such that $h$ is a $C^{\infty}$ diffeomorphism on the interior of the interval. For $f \in T C$ let $\mu_{f}$ denote the pullback by $h$ of the acip (actually Lebesgue measure) for $T$. For $f \in T C, \mu_{f}$ is the measure of maximal entropy. If $f \in T C^{\infty}$ then $\mu_{f}$ is also absolutely continuous.

Then there exist

1. $f \in T C^{\infty}$ with poles at the boundary such that the Lyapunov exponent of $\mu_{f}$ is 2;

2. $f \in T C^{\infty}$ such that the Lyapunov exponent of $\mu_{f}$ does not exist;

3. $f \in T C^{\infty}$ such that $f$ has a smooth parabolic fixed point at the boundary and the Lyapunov exponent of $\mu_{f}$ is 2 ; 
4. $f \in T C$ such that $\mu_{f}$ has positive infinite Lyapunov exponent.

For each $\alpha>0$ let $h_{\alpha}$ be a $C^{\infty}$ homeomorphism of $[0,1]$ with the following properties:

- $\left|D h_{\alpha}\right|>0$ except at 0 and at 1 ;

- on a neighbourhood of 0 one has $h_{\alpha}(x)=e^{-x^{-\alpha}}$;

- the graph of $h$ has a point of central symmetry at $\left(\frac{1}{2}, \frac{1}{2}\right)$, in other words $h(1-x)=1-h(x)$.

For each $\alpha>0$ define $g_{\alpha}:[0,1] \rightarrow[0,1]$ by

$$
g_{\alpha}(x):=h_{\alpha} \circ T \circ h_{\alpha}^{-1} .
$$

Then $g_{\alpha}$ is conjugate to the tent map, symmetric and has non-zero, non-infinite derivative everywhere except for at $0, \frac{1}{2}$, and 1 (figure 2). Denote $\frac{1}{2}$ by $c$. Of course, $g(0)=0, g(c)=1, g(1)=0$. Let us calculate the derivative near 0 . Firstly note that, near zero, $h^{-1}(x)=(-\log x)^{-\frac{1}{\alpha}}$, so

$$
D h^{-1}(x)=\frac{1}{\alpha}(-\log x)^{\frac{-1-\alpha}{\alpha}} \frac{1}{x} .
$$

Clearly $|D T|=2$. Next, $D h(x)=\alpha x^{-1-\alpha} e^{-x^{-\alpha}}$. Putting these together using the chain rule,

$$
\begin{aligned}
D g_{\alpha}(x) & =D h\left(2 h^{-1}(x)\right) 2 D h^{-1}(x) \\
& =\frac{\alpha}{\alpha} 2^{-1-\alpha}(-\log x)^{\frac{1+\alpha}{\alpha}} e^{-2^{-\alpha}(-\log x)^{1}} 2(\log x)^{\frac{-1-\alpha}{\alpha}} \frac{1}{x} \\
& =2^{-\alpha} x^{2^{-\alpha}} \frac{1}{x} \\
& =2^{-\alpha} x^{2^{-\alpha}-1} .
\end{aligned}
$$

One can double-check using the conjugacy that near zero, $g_{\alpha}(x)=x^{2^{-\alpha}}$. The derivative near 1 is the same but with negative sign. We shall see soon that there is a transition in behaviour at $\alpha=1$ which corresponds to a cusp at 0 of root type $=\frac{1}{2}$.

Now let us look at the derivative near the critical point. Here $D h_{\alpha} \neq 0$ and we can assume it is almost constant $\approx D h_{\alpha}(c)=: l^{-1}$, say. If $x$ is close to $c$ then $h_{\alpha}^{-1}(c)$ is close to $\frac{1}{2}$ too, so it gets mapped by $T$ to a point close to 1 . We get

$$
\begin{aligned}
\left|D g_{\alpha}(x+c)\right| & =\left|D h_{\alpha}\left(T h_{\alpha}^{-1}(x+c)\right) 2 D h_{\alpha}^{-1}(x+c)\right| \\
& \approx D h_{\alpha}(2 l|x|) 2 l \\
& =\alpha(2 l|x|)^{-1-\alpha} e^{-(2 l|x|)^{-\alpha}} .
\end{aligned}
$$

We have seen in Corollary 3, that at $\alpha=1$ there is some sort of a transition in other maps with this critical behaviour (polynomial times $e^{-|y|^{-\alpha}}$ ). 
Lebesgue measure is both an acip and a measure of maximal entropy for $T$. We can pull this back by $h_{\alpha}$ to get the measure of maximal entropy for $g_{\alpha}$. This measure $\mu$ is absolutely continuous since $h_{\alpha}$ is smooth and is given by $d \mu(x)=D h_{\alpha}^{-1}(x) d x$. Let us try integrating $\log \left|D g_{\alpha}\right|$ near 0 :

$$
\begin{gathered}
\int \log \left|D g_{\alpha}(x)\right| D h^{-1}(x) d x \\
\approx \int\left(\log 2^{-\alpha}+\left(2^{-\alpha}-1\right) \log x\right)\left(\frac{1}{\alpha}(-\log x)^{\frac{-1-\alpha}{\alpha}} \frac{1}{x}\right) d x \\
=\int \log 2^{-\alpha} D h^{-1}(x)-\left(2^{-\alpha}-1\right) \frac{1}{\alpha}(-\log x)^{\frac{-1}{\alpha}} \frac{1}{x} d x \\
=C_{0}+\int C_{1} \frac{1}{x(-\log x)^{\frac{1}{\alpha}}} d x,
\end{gathered}
$$

where $C_{0}$ and $C_{1}$ are positive constants. This last integral, well known in France as an intégrale de Bertrand, is finite if and only if $\alpha<1$.

Near $c$, since $D h$ is approximately a positive constant, $\log \left|D g_{\alpha}\right|$ will be integrable with respect to $\mu$ if and only if it integrable with respect to Lebesgue. It is easy to see that it is integrable with respect to Lebesgue if and only if $\alpha<1$.

Thus $g_{\alpha}$ has an absolutely continuous invariant probability measure with finite Lyapunov exponent if $\alpha<1$. In this case, since $h_{\alpha}$ is smooth everywhere bar the boundary, every periodic point $p$ of period $k$ satisfies $\left|D f^{k}(p)\right|=2^{k}$, so the Lyapunov exponent is equal to 2.

If $\alpha \geq 1$ then $g_{\alpha}$ has an acip whose Lyapunov exponent does not exist. At the critical point $\int \log \left|D g_{\alpha}\right| d \mu$ is $-\infty$; at 0 it is $+\infty$. If we write $\underline{\chi}(x)$ and $\bar{\chi}(x)$ for the lower and upper Lyapunov exponents of $g_{\alpha}$ at $x$, then, almost everywhere,

$$
\underline{\chi}(x) \leq 2 \leq \bar{\chi}(x) .
$$
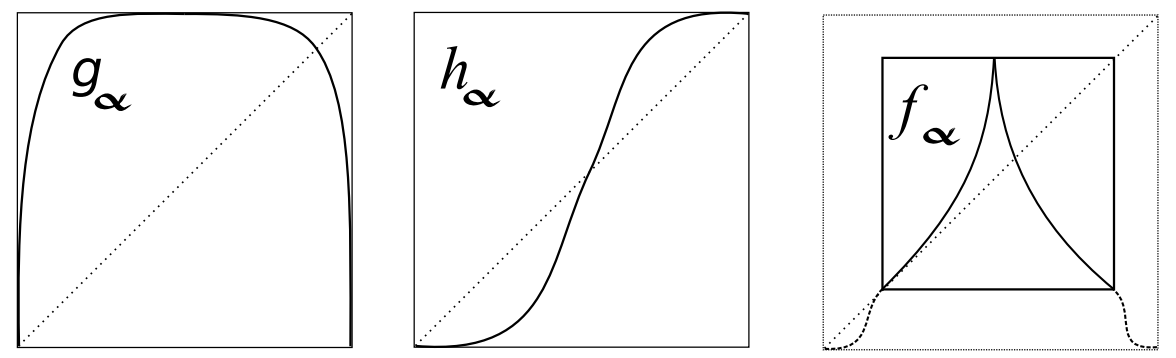

Figure 2: Graphs of $g_{\alpha}, h_{\alpha}$ and $f_{\alpha}$.

Now we conjugate in the other direction. For each $\alpha>0$ define $f_{\alpha}:[0,1] \rightarrow$ $[0,1]$ by

$$
f_{\alpha}(x):=h_{\alpha}^{-1} \circ T \circ h_{\alpha} .
$$


Again, $f_{\alpha}$ is symmetric, conjugate to the tent map and the derivative is non-zero and finite except possibly at $0, c, 1$. In fact the norm of the derivative is strictly positive and finite everywhere except for at $c$ (figure 21). Let us calculate $D f_{\alpha}$ near 0 .

$$
\begin{aligned}
D f_{\alpha}(x) & =D h_{\alpha}^{-1}\left(2 e^{-x^{-\alpha}}\right) 2 \alpha x^{-\alpha-1} e^{-x^{-\alpha}} \\
& =\alpha^{-1}\left(-\log 2+x^{-\alpha}\right)^{\frac{-1-\alpha}{\alpha}} \frac{1}{2} e^{x^{-\alpha}} 2 \alpha x^{-\alpha-1} e^{-x^{-\alpha}} \\
& =\left(-\log 2+x^{-\alpha}\right)^{\frac{-1-\alpha}{\alpha}} x^{-\alpha-1} \\
& =\left(1-x^{\alpha} \log 2\right)^{\frac{-1-\alpha}{\alpha}} .
\end{aligned}
$$

Thus $D f_{\alpha}(0)=1$ and there is a parabolic fixed point at zero. It is straightforward to check that, for all $i$ such that $2 \leq i<\alpha+1, D^{i} f_{\alpha}(0)$ exists and $D^{i} f_{\alpha}(0)=0$. Also, $f_{\alpha}$ is $C^{\infty}$ near zero if and only if $\alpha>0$ is a natural number, in which case $D^{\alpha+1} f_{\alpha}(0)>0$.

Near the critical point we have

$$
\begin{aligned}
\left|D f_{\alpha}(c+x)\right| & =\left|D h_{\alpha}^{-1}\left(T h_{\alpha}(x+c)\right) 2 D h_{\alpha}(x+c)\right| \\
& \approx D h_{\alpha}^{-1}\left(2 l^{-1}|x|\right) 2 l^{-1} \\
& =2 l^{-1} \alpha^{-1}\left(-\log \left(l^{-1} 2|x|\right)\right)^{\frac{-1-\alpha}{\alpha}} 2^{-1} l|x|^{-1} \\
& =\alpha^{-1}|x|^{-1}\left(-\log \left(l^{-1} 2|x|\right)\right)^{\frac{-1-\alpha}{\alpha}} .
\end{aligned}
$$

Hence the derivative does indeed tend to infinity at $c$. Moreover, $\frac{1}{D f_{\alpha}}$ is $C^{\epsilon}$ near $c$, so an extension of $f_{\alpha}$ is an cusp map.

Pulling back Lebesgue measure, we get an absolutely continuous invariant probability measure $\mu$ for $f_{\alpha}$ with $C^{\infty}$ density $D h(x)$, despite the existence of a smooth parabolic point in the support of $\mu$. The Lyapunov exponent here is integrable and equal to 2 .

Now we show that there is a cusp map $f$ in $T C$ whose measure of maximal entropy has infinite Lyapunov exponent. Let $f:[0,1] \rightarrow[0,1]$ be a map in $T C$ with poles at the boundary and at the preimage $c$ of the turning point $\frac{1}{2}$, such that on $U$ a neighbourhood of zero, $f(x)=x^{\alpha}$ for some $\alpha$ with $0<\alpha<1$. See figure 3 .

Let $f_{1}:=f_{\mid[0, c]}$ and let $p, N$ be such that $p=f_{1}^{-N}(c) \in U$ and set $p_{n}:=$ $f_{1}^{-n}(p)$. Then

$$
p_{n}=p^{\frac{1}{\alpha^{n}}} .
$$

On $\left[p_{n+1}, p_{n}\right]$ one has $\alpha p_{n+1}^{\alpha-1} \leq D f \leq \alpha p_{n}^{\alpha-1}$, and $\mu_{f}\left(\left[p_{n+1}, p_{n}\right]=2^{-(n+N+1)}\right.$ since $f$ is conjugate to the full tent map $T_{2}$. So, on $\left[p_{n+1}, p_{n}\right]$,

$$
\log \alpha+(\alpha-1) \frac{1}{\alpha^{n+1}} \log p \leq \log D f \leq \log \alpha+(\alpha-1) \frac{1}{\alpha^{n}} \log p
$$


Subtracting $\log \alpha$ and integrating,

$$
\begin{aligned}
(\alpha-1) \log p \sum_{i=0}^{\infty} \frac{1}{\alpha^{i+1}} 2^{-(i+N+1)} & \leq-\mu_{f}([0, p]) \log \alpha+\int_{0}^{p} \log D f d \mu_{f} \\
& \leq(\alpha-1) \log p \sum_{i=0}^{\infty} \frac{1}{\alpha^{i}} 2^{-(i+N+1)}
\end{aligned}
$$

so, near zero, $\int \log D f d \mu_{f}$ is finite if $\alpha>\frac{1}{2}$ and positive infinite if $\alpha \leq \frac{1}{2}$. By definition, the derivative is bounded away from zero, so, if $\alpha \leq \frac{1}{2}$, then

$$
\int_{[0,1]} \log |D f| d \mu=+\infty
$$

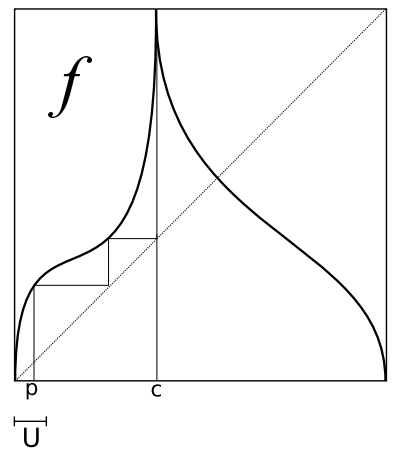

Figure 3: Graph of $f$.

\section{Acknowledgments}

The author would like to thank his former supervisor Jacek Graczyk for his encouragement and for many enlightening conversations. Most of the present work was carried out while a doctoral student at Université Paris-Sud. The author is currently a postdoctoral researcher at the University of Helsinki. The author has benefited from funding from a Goldstine Fellowship (IBM), the Knut and Alice Wallenberg Foundation, the Göran Gustafsson Foundation and the EU Training Network CODY. Careful reading by a referee led to substantial improvement of this paper, for which the author is very grateful.

\section{References}

[1] Vítor Araújo, Stefano Luzzatto, and Marcelo Viana. Invariant measures for interval maps with critical points and singularities. Adv. Math., 221(5):1428-1444, 2009. 
[2] Magnus Aspenberg. Rational Misiurewicz maps are rare. Comm. Math. Phys., 291(3):645-658, 2009.

[3] Michael Benedicks and Michał Misiurewicz. Absolutely continuous invariant measures for maps with flat tops. Inst. Hautes Études Sci. Publ. Math., (69):203-213, 1989.

[4] A. M. Blokh and M. Yu. Lyubich. Measurable dynamics of $S$-unimodal maps of the interval. Ann. Sci. École Norm. Sup. (4), 24(5):545-573, 1991.

[5] H. Bruin. Induced maps, Markov extensions and invariant measures in one-dimensional dynamics. Comm. Math. Phys., 168(3):571-580, 1995.

[6] H. Bruin, J. Rivera-Letelier, W. Shen, and S. van Strien. Large derivatives, backward contraction and invariant densities for interval maps. Invent. Math., 172(3):509-533, 2008.

[7] Henk Bruin, Weixiao Shen, and Sebastian van Strien. Invariant measures exist without a growth condition. Comm. Math. Phys., 241(2-3):287-306, 2003.

[8] Henk Bruin and Mike Todd. Equilibrium states for interval maps: the potential $-t \log |D f|$. Ann. Sci. Éc. Norm. Supér. (4), 42(4):559-600, 2009.

[9] Welington de Melo and Sebastian van Strien. One-dimensional dynamics, volume 25 of Ergebnisse der Mathematik und ihrer Grenzgebiete (3) [Results in Mathematics and Related Areas (3)]. Springer-Verlag, Berlin, 1993.

[10] K. Díaz-Ordaz, M. P. Holland, and S. Luzzatto. Statistical properties of one-dimensional maps with critical points and singularities. Stoch. Dyn., 6(4):423-458, 2006.

[11] Neil Dobbs. Critical points, cusps and induced expansion in dimension one. Doctoral thesis, Université Paris-Sud, 2006.

[12] Neil Dobbs. Visible measures of maximal entropy in dimension one. Bull. Lond. Math. Soc., 39(3):366-376, 2007.

[13] Neil Dobbs. Measures with positive Lyapunov exponent and conformal measures in rational dynamics. Trans. Amer. Math. Soc., 364(6):2803$2824,2012$.

[14] Neil Dobbs and Bartłomiej Skorulski. Non-existence of absolutely continuous invariant probabilities for exponential maps. Fund. Math., 198(3):283$287,2008$.

[15] Jacek Graczyk, Duncan Sands, and Grzegorz Świạtek. Metric attractors for smooth unimodal maps. Ann. of Math. (2), 159(2):725-740, 2004.

[16] Franz Hofbauer. On intrinsic ergodicity of piecewise monotonic transformations with positive entropy. Israel J. Math., 34(3):213-237 (1980), 1979. 
[17] Franz Hofbauer. On intrinsic ergodicity of piecewise monotonic transformations with positive entropy. II. Israel J. Math., 38(1-2):107-115, 1981.

[18] Franz Hofbauer and Peter Raith. The Hausdorff dimension of an ergodic invariant measure for a piecewise monotonic map of the interval. Canad. Math. Bull., 35(1):84-98, 1992.

[19] Franz Hofbauer and Peter Raith. The Hausdorff dimension of an ergodic invariant measure for a piecewise monotonic map of the interval. Canad. Math. Bull., 35(1):84-98, 1992.

[20] Gerhard Keller. Lifting measures to Markov extensions. Monatsh. Math., 108(2-3):183-200, 1989.

[21] Gerhard Keller. Exponents, attractors and Hopf decompositions for interval maps. Ergodic Theory Dynam. Systems, 10(4):717-744, 1990.

[22] François Ledrappier. Some properties of absolutely continuous invariant measures on an interval. Ergodic Theory Dynamical Systems, 1(1):77-93, 1981.

[23] François Ledrappier. Quelques propriétés ergodiques des applications rationnelles. C. R. Acad. Sci. Paris Sér. I Math., 299(1):37-40, 1984.

[24] Stefano Luzzatto and Warwick Tucker. Non-uniformly expanding dynamics in maps with singularities and criticalities. Inst. Hautes Études Sci. Publ. Math., (89):179-226 (2000), 1999.

[25] Stefano Luzzatto and Marcelo Viana. Positive Lyapunov exponents for Lorenz-like families with criticalities. Astérisque, (261):xiii, 201-237, 2000. Géométrie complexe et systèmes dynamiques (Orsay, 1995).

[26] Marco Martens. Distortion results and invariant Cantor sets of unimodal maps. Ergodic Theory Dynam. Systems, 14(2):331-349, 1994.

[27] Sheldon E. Newhouse. Entropy and volume. Ergodic Theory Dynam. Systems, 8*(Charles Conley Memorial Issue):283-299, 1988.

[28] William Parry. Topics in ergodic theory, volume 75 of Cambridge Tracts in Mathematics. Cambridge University Press, Cambridge, 1981.

[29] V. A. Rohlin. Exact endomorphisms of a Lebesgue space. Amer. Math. Soc. Transl. (2), 39:1-36, 1964.

[30] David Ruelle. An inequality for the entropy of differentiable maps. Bol. Soc. Brasil. Mat., 9(1):83-87, 1978.

[31] Marek Rychlik. Bounded variation and invariant measures. Studia Math., 76(1):69-80, 1983. 
[32] Duncan Sands. Misiurewicz maps are rare. Comm. Math. Phys., 197(1):109-129, 1998.

[33] Hans Thunberg. Positive exponent in families with flat critical point. Ergodic Theory Dynam. Systems, 19(3):767-807, 1999.

[34] Roland Zweimüller. $S$-unimodal Misiurewicz maps with flat critical points. Fund. Math., 181(1):1-25, 2004. 